\title{
"1-N" Leader-Follower Formation Control of Multiple Agents Based on Bearing-Only Observation
}

\author{
Qing $\operatorname{Han}^{1}$, Tiancheng $\mathrm{Li}^{1(\varangle)}$, Shudong Sun ${ }^{1}$, \\ Gabriel Villarrubia ${ }^{2}$, and Fernando de la Prieta ${ }^{2}$ \\ ${ }^{1}$ School of Mech. Eng., Northwestern Polytechnical University, Xi' an 710072, China \\ \{hanqing, t.c.li\} @mail.nwpu.edu.cn \\ ${ }^{2}$ Faculty of Science, University of Salamanca, 37008, Salamanca, Spain \\ \{gvg, fer\}@usal.es
}

\begin{abstract}
A formation control method is proposed for multiple agents of "1" leader and " $\mathrm{N}$ " follower where the following $\mathrm{N}$-agents (as called followers) can only observe the bearing information of the leading 1-agent (as called the leader). It is proven that bearing-only observation meets the observability condition required for the "1-N" leader-follower formation system. The unscented Kalman filter is employed to estimate the relative position of the leader, based on which the input-output feedback control law is executed to control the real-time movement of the followers so that the " $1-\mathrm{N}$ " leader-followers formation is properly maintained. Simulation results demonstrate the effectiveness of our approach.
\end{abstract}

Keywords: Multi-agent system $\cdot$ Formation control $\cdot$ Unscented Kalman filter

\section{Introduction}

Multi-agent systems have matured during the last decade and many effective applications have been deployed in terms of both software and hardware (Carrascosa et al. 2008, Zato et al. 2012). This papers concerns on the formation control of multi-agent of the "1-N" structure where several following agents (as called followers) are selfcontrolled to follow a leading agent (leader) based on the bearing-only observation from the followers to the leader. That is, the motion of the leader defines the desired motion, while the followers are controlled to follow it. This is particularly related to the multi-robot formation, which is of high interest to underwater or outer space exploration, shop floor transportation, guarding, escorting, and patrolling missions. A variety of formation control methods have been proposed, such as virtual structure approach (Ren et al. 2004), leader-follower approach (Sun et al. 2012; Chen et al. 2009; Shao et al. 2007; Consolini et al. 2008; Cristescu et al. 2012), artificial potential (Kwon 2012), graph theory (Sharma et al. 2012). Among them, the leader-follower formation that consists of " 1 " leader and " $N$ " followers has been mostly used owing to its universality, scalability and reliability.

The formation control becomes challenging when the observation information between agents is poor. Most of the existing leader-follower approaches as mentioned use both distance and bearing observation. However, in many real-life situations, 
available observations might be only the bearing information, namely bearing-only formation control, which will pose a great challenge to the formation control. This challenging albeit important problem has been executed in Moshtagh et al. (2008), Basiri et al. (2010), Bishop (2011a, 2011b) and Eren (2012) based on special requirements on the number of agents or the form of the formation. A distributed control law is used in Franchi et al. (2012) and Zhao et al. (2014a) to stabilize the formations, based on which angle constraint is further considered in Zhao et al. (2014b). In Mariottini et al. (2009), the observability condition for position estimation by using bearing-only observations is estiblished. In order to utilize the feedback control law, an "off-the-axis" point has to be constructed in Mariottini et al. (2009), Morbidi et al. (2010) and Das et al. (2002). Differently, our approach will rely on neither special requirement for the form of the formation and nor off-the-axis point.

In particular, position estimation of each agent is critical to generate the real time form information that is required to control the movement of the follower. Estimation algorithms available include the extended Kalman filter (EKF) (Mariottini et al. 2009), the extended information filter (EIF) (Sharma et al.2013), the particle filter (PF) (Li et al. 2010) and maximum a posteriori (MAP) (Nerurkaret al. 2009). Most of them except the PF are not well qualified to deal with highly nonlinear systems while the PF is very computationally intensive. In contrast, the UKF sits between them, which handles nonlinearities with higher accuracy than the EKF and requires lesser computational requirement than the PF.

The contribution of this paper is two-fold. Firstly, the observability of the "1-N" multi-agent formation is studied based on the rank of the observability matrix (Section 2). Secondly, an UKF based on bearing-only observations is designed for the state estimation of multi-agents, rendering real-time and reliable movement control of the followers via the input-output feedback control law (Section 3) which gets rid of off-the-axis points. Properly designed simulations are given (Section 4) to demonstrate the validity of our approach. We offer our conclusions (Section 5).

\section{Problem Statement and Observability Analysis}

\subsection{Problem Statement}

This section will formulate the " $1-\mathrm{N}$ " bearing-only observation model and give the notations used. As shown in Fig. $1, R_{1}$ represents the leader while $R_{2}$ is a follower (for simplicity, we only show one here). The control inputs for agents are linear and

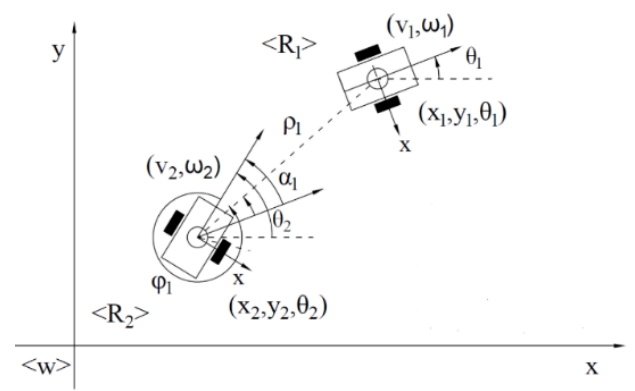

Fig. 1. 1-1 Leader-Follower coordinate definition 
angular velocities $\left[v_{i}, w_{i}\right], i=1,2, \ldots, \rho_{i}$ is the distance from the centroid of the leader to the centroid of the follower. $\varphi_{i}$ is the view-angle from the $y$-axis of the follower to the centroid of the leader-robot. $\theta_{i}$ and $\theta_{j}$ are the orientations of the leader and the follower with respect to the world frame $\langle\mathrm{W}\rangle$, respectively, while $\alpha_{i}$ is the relative orientation between the leader and the follower robot, i.e., $\alpha_{i} \triangleq \theta_{i}-\theta_{j}$.

With reference to Fig. 1, the kinematic model of a 1-1(one leader and one follower) formation can be expressed as follows

$$
S_{n}:\left\{\begin{array}{c}
\dot{\boldsymbol{s}}=f(s, u)=F(s) U \\
\boldsymbol{y}=h(s)=\left[h_{1}^{\mathrm{T}}(s)\right]^{\mathrm{T}}=\left[\begin{array}{ll}
\varphi_{1} & \alpha_{1}
\end{array}\right]^{\mathrm{T}}
\end{array}\right.
$$

where state vector $\mathbf{s} \triangleq\left[s_{1}^{\mathrm{T}}\right]^{\mathrm{T}}, s_{1} \triangleq\left[\rho_{1} \varphi_{1} \alpha_{1}\right]^{\mathrm{T}}, \delta_{1}=\varphi_{1}+\alpha_{1}$, input vector $\mathbf{u} \triangleq$ $\left[\begin{array}{llll}v_{1} & w_{1} & v_{2} & w_{2}\end{array}\right]^{\mathrm{T}}$, output vector $\mathbf{y}=h(s)=\left[h_{1}^{\mathrm{T}}\right]^{\mathrm{T}}, h_{1} \triangleq\left[\begin{array}{ll}\varphi_{1} & \alpha_{1}\end{array}\right]^{\mathrm{T}}$ and

$$
F(s)=\left[\begin{array}{cccc}
\cos \delta_{1} & 0 & -\cos \varphi_{1} & 0 \\
-\sin \delta_{1} / \rho_{1} & 0 & \sin \varphi_{1} / \rho_{1} & -1 \\
0 & 1 & 0 & -1
\end{array}\right]
$$

The kinematic model of the " $1-\mathrm{N}$ " (one leader and $n$ follower) formation can be readily retrieved as an extension of (1),

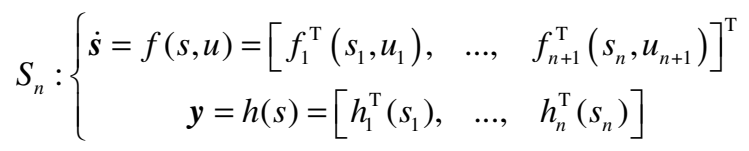

where $s=\left[s_{1}^{\mathrm{T}}, s_{2}^{\mathrm{T}}, \cdots, s_{n}^{\mathrm{T}}\right]^{\mathrm{T}} \in \mathbb{R}^{3 n}, U=\left[u_{1}^{\mathrm{T}}, u_{2}^{\mathrm{T}}, \cdots, u_{n+1}^{\mathrm{T}}\right]^{\mathrm{T}} \in \mathbb{R}^{2(n+1)}, h: \mathbb{R}^{2 n} \mapsto \mathbb{R}^{2 n}$, and $f: \mathbb{R}^{3(n+1)} \times \Lambda \mapsto \mathbb{R}^{3(n+1)}$.

To make the formation control problem solvable, the system must be observable. The system is defined to be observable only if the system output convey an information that is sufficient to allow the follower to obtain correct estimates of the position of the leader; otherwise, the system is not observable. In the following, we will analysis the observability of the "1-N" leader-followers system based on the nonlinear observability rank criteria that is developed in Hermann et al. (1977).

The observability matrix of (2) is defined as

$$
M=\left[\nabla L_{f_{v_{i}}, \cdots, f_{v_{j}}, f_{\omega_{i}}, \cdots f_{\omega_{j}}}^{p} h_{k}(s)\right]
$$

where $i=1, k=1, \cdots, n, j=2, \cdots, n+1, p \in \mathbb{N}, \nabla$ represents the gradient operator.

Lemma 1: System $S_{n}$ is (locally weakly) observable if its observability matrix $M$ whose rows are given in (3) has full rank (Hermann et al. 1977), e.g., in our case $\operatorname{rank}(M)=3 n, n$ refers to the number of followers.

\subsection{Bearing-Only Observability}

We analysis the observability based on Lemma 1. For simplicity, we only analysis the 1-1 system of one leader and one follower. This result is readily extendable to the 
parallel " $1-\mathrm{N}$ " system of one leader and multiple followers, since each follower is independent to each other. The function $f(\cdot)$ can be separated into a summation of independent functions in the special case, and each one excited by a different component of the control input vector, (1) can be restated as follows

$$
S_{n}:\left\{\begin{array}{c}
\dot{\boldsymbol{s}}=f(s, u)=f_{v_{1}} v_{1}+f_{w_{1}} \omega_{1}+f_{v_{2}} v_{2}+f_{w_{2}} \omega_{2} \\
\boldsymbol{y}=h(s)=\left[h_{1}^{\mathrm{T}}(s)\right]^{\mathrm{T}}=\left[\begin{array}{ll}
\varphi_{1} & \alpha_{1}
\end{array}\right]^{\mathrm{T}}
\end{array}\right.
$$

where,

$$
\begin{gathered}
f_{v_{1}}=\left[\begin{array}{ccc}
\cos \delta_{1} & -\sin \delta_{1} / \rho_{1} & 0
\end{array}\right]^{\mathrm{T}} \\
f_{\omega_{1}}=\left[\begin{array}{lll}
0 & 0 & 1
\end{array}\right]^{\mathrm{T}} \\
f_{v_{2}}=\left[\begin{array}{ccc}
-\cos \varphi_{1} & \sin \varphi_{1} / \rho_{1} & 0
\end{array}\right]^{\mathrm{T}} \\
f_{\omega_{2}}=\left[\begin{array}{lll}
0 & -1 & -1
\end{array}\right]^{\mathrm{T}}
\end{gathered}
$$

Proposition 2 given in Morbidi et al. (2010) indicates that the gradients of the Lie derivatives of $h(s)$ are equal to the same order gradients of the time derivatives of $h(s)$ from. That is, the zeroth-order Lie derivative is:

$$
L^{0} h_{1}(s)=h_{1}(s)=\left[\begin{array}{ll}
\varphi_{1} & \alpha_{1}
\end{array}\right]^{\mathrm{T}}
$$

and gradient is,

$$
\nabla L^{0} h_{1}(s)=\left[\begin{array}{lll}
0 & 1 & 0 \\
0 & 0 & 1
\end{array}\right]
$$

The first-order Lie derivative of the function $h_{1}(s)$ with respect to $f_{v}$ and $f_{\omega}$ are defined as follows,

$$
\begin{gathered}
L_{f_{v_{1}}}^{1} h_{1}(s)=\nabla\left[L^{0} h_{1}(s)\right] \cdot f_{v_{1}}=\left[\begin{array}{ll}
-\sin \left(\delta_{1}\right) / \rho_{1} & 0
\end{array}\right]^{\mathrm{T}} \\
L_{f_{\omega_{1}}}^{1} h_{1}(s)=\nabla\left[L^{0} h_{1}(s)\right] \cdot f_{\omega_{1}}=\left[\begin{array}{ll}
0 & 1
\end{array}\right]^{\mathrm{T}} \\
L_{f_{v_{2}}}^{1} h_{1}(s)=\nabla\left[L^{0} h_{1}(s)\right] \cdot f_{v_{2}}=\left[\begin{array}{ll}
\sin \left(\varphi_{1}\right) / \rho_{1} & 0
\end{array}\right]^{\mathrm{T}} \\
L_{f_{\omega_{2}}}^{1} h_{1}(s)=\nabla\left[L^{0} h_{1}(s)\right] \cdot f_{\omega_{2}}=\left[\begin{array}{ll}
-1 & -1
\end{array}\right]^{\mathrm{T}}
\end{gathered}
$$

where "." denotes the vector inner product, with their gradients given as follows

$$
\begin{gathered}
\nabla L_{f_{v_{1}}}^{1} h_{1}(s)=\left[\begin{array}{ccc}
\sin \left(\delta_{1}\right) / \rho_{1}^{2} & -\cos \left(\delta_{1}\right) / \rho_{1} & -\cos \left(\delta_{1}\right) / \rho_{1} \\
0 & 0 & 0
\end{array}\right] \\
\nabla L_{f_{\omega_{1}}}^{1} h_{1}(s)=\left[\begin{array}{lll}
0 & 0 & 0 \\
0 & 0 & 0
\end{array}\right] \\
\nabla L_{f_{v_{2}}}^{1} h_{1}(s)=\left[\begin{array}{ccc}
-\sin \left(\varphi_{1}\right) / \rho_{1}^{2} & \cos \left(\varphi_{1}\right) / \rho_{1} & 0 \\
0 & 0 & 0
\end{array}\right] \\
\nabla L_{f_{\omega_{2}}}^{1} h_{1}(s)=\left[\begin{array}{lll}
0 & 0 & 0 \\
0 & 0 & 0
\end{array}\right]
\end{gathered}
$$

Substituting the above gradients of Lie derivatives into the observability matrix (3), we have 


$$
M=\left[\begin{array}{c}
\nabla L^{0} h_{1}(s) \\
\nabla L_{f_{v_{1}}}^{1} h_{1}(s) \\
\nabla L_{f_{\omega_{1}}}^{1} h_{1}(s) \\
\nabla L_{f_{v_{2}}}^{1} h_{1}(s) \\
\nabla L_{f_{\omega_{2}}}^{1} h_{1}(s)
\end{array}\right]=\left[\begin{array}{rrl}
0_{2 \times 1} & I_{2 \times 2} & \\
\sin \left(\delta_{1}\right) / \rho_{1}^{2} & -\cos \left(\delta_{1}\right) / \rho_{1} & -\cos \left(\delta_{1}\right) / \rho_{1} \\
0_{3 \times 3} & \\
-\sin \left(\varphi_{1}\right) / \rho_{1}^{2} & \cos \left(\varphi_{1}\right) / \rho & 0 \\
0_{3 \times 3} &
\end{array}\right]
$$

It shows that the rank of the observability matrix does not change with the increasing order of the Lie derivatives. The observability matrix can be determined based on the gradients of the first-order and zeroth order Lie derivatives. Then, we have the following two remarks on the observability of the bearing-only leader-follower system as described so far.

Remark 1: $\operatorname{rank}(M)=3$ if

1) $v_{1}>0, v_{j}>0$, where $j=2, \cdots, n+1$;

2) $\varphi_{i} \neq 0, i=1, \cdots, n$;

3) The leader and the follower move neither in parallel nor straightly.

Proof: Given the above three prerequisite requirements, the $M$ matrix by means of a finite sequence of elementary row operations, can be transformed to a simplified form as given in (20), obtaining $\operatorname{rank}(M)=3$.

Remark 2: $\operatorname{rank}(M)=2$ if

$$
M \Rightarrow\left[\begin{array}{ccc}
1 & 0 & 0 \\
0 & 1 & 0 \\
0 & 0 & 1 \\
& O_{7 \times 3} &
\end{array}\right]
$$

1) $v_{1}>0, v_{j}>0$, where $j=2, \cdots, n+1$;

2) $\varphi_{i}=k_{1}, \alpha_{i}=k_{2}$, where $i=1, \cdots, n$, and $k_{1}, k_{2}$ is constant, i.e., the leader and the followers move straightly (in parallel).

Proof: Supposing $\varphi_{i}=k_{1}, \alpha_{i}=k_{2}, k_{1}, k_{2}$ are constant and the differentiation of the functions $\varphi_{i}$ and $\alpha_{i}$ are zero, we have

$$
\begin{gathered}
\nabla h_{1}^{(0)}(s)=\left[\begin{array}{lll}
0 & 1 & 0 \\
0 & 0 & 1
\end{array}\right] \\
h_{1}^{(1)}(s)=h_{1}^{(2)}(s)=\cdots=h_{1}^{(n-1)}(s)=0 \\
\nabla h_{1}^{(1)}(s)=\nabla h_{1}^{(2)}(s)=\cdots=\nabla h_{1}^{(n-1)}(s)=\left[\begin{array}{lll}
0 & 0 & 0 \\
0 & 0 & 0
\end{array}\right]
\end{gathered}
$$

These lead to a simplified form of $M$ matrix as follows

$$
M \Rightarrow\left[\begin{array}{ccc}
0 & 1 & 0 \\
0 & 0 & 1 \\
& O_{8 \times 3} &
\end{array}\right]
$$


i.e. $\operatorname{rank}(M)=2$.

Based on Remark 1 and 2, we have the conclusion that $M$ has full rank (that the system is locally weakly observable) when the agents move along a curvilinear line (moving in neither parallel nor straightly) and rank $(M)=2$ when the agent move along a straight line (the system is not locally observable).

\section{UKF-Based Input-Output Feedback Control}

To maintain a desired "1-N" formation, the followers need to know the relative position of the leader, and adjusts their current positions accordingly in real time. In our approach, the former is estimated by UKF while the latter is carried out based on the classical input-output feedback control law. In the following, we will explain how to calculate the control input required for the followers for formation.

To implement UKF, the continuous-time state dynamic (1) needs to be discretized firstly. Assume both the continuous-time state dynamics (25) and the observation equation (26) are affected with additive noises as

$$
\begin{aligned}
& \dot{\boldsymbol{s}}=F(s) U+\mathrm{O} \\
& \boldsymbol{y}=G s+N
\end{aligned}
$$

where $G$ is the output transition matrix, $O$ and $N$ are white Gaussian noises with zero mean and covariance matrices $P_{O}$ and $P_{N}$, respectively. We assume that $s(0), O$ and $N$ are uncorrelated for simplicity. We apply the Euler forward method with sampling time $T_{c}$ to discretize the state dynamics (25), obtaining

$$
s(k+1)=\Gamma(s(k), u(k))+T_{c} O
$$

where $\Gamma(s(k), u(k))=T_{c} F(s) U+s(k)$ and $k \in \mathbb{N}$.

By taking the derivative on $\alpha_{1} \triangleq \theta_{1}-\theta_{2}$, we have

$$
\begin{aligned}
& \dot{\boldsymbol{s}}_{r}=L(s) U_{1}+M(s) U_{2} \\
& \dot{\alpha}_{1}=\omega_{1}-\omega_{2}
\end{aligned}
$$

where $s_{r} \triangleq\left[\rho_{1} \varphi_{1}\right]^{\mathrm{T}}$ is the reduced state-space vector. $L^{2 \times 2}$ and $M^{2 \times 2}$ are the upperleft and right submatrices of $F$, respectively.

The input-output feedback control law (Slotine et al. 1991, Das et al. 2002) algebraically linearizes a nonlinear system dynamics and then calculates the control put $U$ for the follower to achieve a desired $s_{r}^{\text {ide }}$.

The control input for our input-output feedback control system is:

$$
\boldsymbol{U}_{2} \triangleq\left[\begin{array}{ll}
v_{2} & \omega_{2}
\end{array}\right]^{\mathrm{T}}=M^{-1}(s)\left(C-L(s) U_{1}\right)
$$

where

$$
C=-K\left(s_{r}-s_{r}^{\text {ide }}\right)
$$

and $K=\operatorname{diag}\left[k_{1} k_{2}\right]$, with $k_{1}, k_{2}>0$, the superscript "ide" refers to the desired state, and $C$ is auxiliary control parameter. Eq. (30) serves as a feedback linearizing control for Eq. (28). Substituting Eq. (30) into Eq. (28), we have 


$$
\dot{\boldsymbol{s}}_{r}=C=-K\left(s_{r}-s_{r}^{\text {ide }}\right)=\left[\begin{array}{l}
k_{1}\left(\rho_{1}^{\text {ide }}-\rho_{1}\right) \\
k_{2}\left(\varphi_{1}^{\text {ide }}-\varphi_{1}\right)
\end{array}\right]
$$

\section{Simulation}

In order to demonstrate the validity of the proposed formation control approach, simulations are designed based on the hybrid platform of Webots 7 and Matlab. The leader-followers formation of four agents is formed where followers $R_{2}, R_{3}$ and $R_{4}$ follow the leader $R_{1}$. The simulation scenario given in Webots 7 is shown in Fig. 2. In the simulation, two typical trajectories are designed for the leader. As stated, to make the system always weakly observable $(\operatorname{rank}(M)=3)$, the trajectories do not include straight and parallel movements for the leader and followers. In scenario 1 (as shown in Fig.3 (a)), the trajectory of the leader is a complete elliptical. In scenario 2 (as shown in Fig.4 (a)), the leader will move straightly (shortly) firstly and then turn right and left in circles. Simulation results are given in subfigures of Fig. 3 and 4 for scenario 1 and 2 respectively.

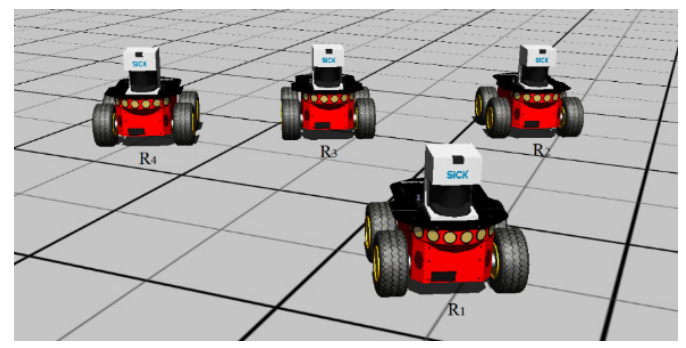

Fig. 2. The simulation scene of mobile agents

\subsection{Simulation Setup}

In scenario 1 , the following velocity inputs are assigned to the leader

$$
v_{1}(t)=2.0 \mathrm{~m} / \mathrm{s}, \omega_{1}(t)=\pi / 5 \mathrm{rad} / \mathrm{s}, t \in[0,10]
$$

while in scenario 2 , the following velocity inputs are assigned to the leader

$$
v_{1}(t)=2.0 \mathrm{~m} / \mathrm{s}, \omega_{1}(t)=\left\{\begin{array}{c}
0 \mathrm{rad} / \mathrm{s}, t \in\{[0,2),[8,9)\} \\
-\pi / 5 \mathrm{rad} / \mathrm{s}, t \in[9,12) \\
\pi / 5 \mathrm{rad} / \mathrm{s}, t \in\{[2,8),[12,14]\}
\end{array}\right.
$$

For both scenarios, the initial vectors of the leader and three followers are

$$
\begin{aligned}
& {\left[\begin{array}{lll}
x_{1}(0) & y_{1}(0) & z_{1}(0)
\end{array}\right]^{\mathrm{T}}=\left[\begin{array}{lll}
0 & 0 & 0
\end{array}\right]^{\mathrm{T}},\left[\begin{array}{lll}
x_{2}(0) & y_{2}(0) & z_{2}(0)
\end{array}\right]^{\mathrm{T}}=\left[\begin{array}{lll}
-0.1 & -0.25 & 0
\end{array}\right]^{\mathrm{T}} \text {, }} \\
& {\left[\begin{array}{lll}
x_{3}(0) & y_{3}(0) & z_{3}(0)
\end{array}\right]^{\mathrm{T}}=\left[\begin{array}{lll}
-0.1 & 0.2 & 0
\end{array}\right]^{\mathrm{T}},\left[\begin{array}{lll}
x_{4}(0) & y_{4}(0) & z_{4}(0)
\end{array}\right]^{\mathrm{T}}=\left[\begin{array}{lll}
-0.1 & 0.4 & 0
\end{array}\right]^{\mathrm{T}} \text {. }}
\end{aligned}
$$

Parameters required for the input-output state feedback control are set follows.

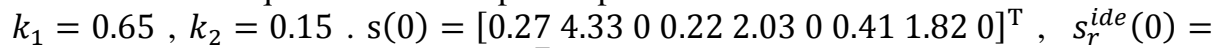

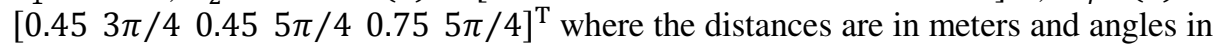
radians. For the parameters required by the UKF, $T_{c}=0.01 \mathrm{~s}, P_{O}=\operatorname{diag}[h \quad h], P_{N}=$ $\operatorname{diag}\left[\begin{array}{ll}h & h\end{array}\right], P=\operatorname{diag}\left[\begin{array}{ll}1.13 & 1.13\end{array}\right]$, where $h=2.11 \times 10^{-2} \mathrm{rad}^{2}$. 


\subsection{Simulation Analysis}

Fig. 3 (a) and 4 (a) give the trajectories of the leader and followers, showing intuitively that the desired formation are properly maintained in both scenarios. This is because the system is observable under the given scenario according to the observability described in Section 2.2. Fig. 3. (b), (c) and (d) present the observation angle estimation error, direction angle estimation error and velocities of the followers respectively. The results show that both the observation and direction angle errors are quite small after the initial stage (the formation becomes stable) and the velocities of the follower agents change slightly during the entire input-output feedback control process. The velocity of the follower agent outside of the leader is larger than the velocity of the leader and inside of the leader is smaller. Similar results are shown in scenario 2. It is shown in Fig. 4. (b) that the observation angle estimation error is very small. Fig. 4. (c) and (d) show that the direction angle estimation error is also very small and the velocities of the follower are relatively stable during the input-output feedback control process. There is an exception. When the leader changes moving direction suddenly, the maximum direction angle error occurs around -0.0126 and the velocities of the followers change significantly. Overall, these results indicate a stable performance of the proposed formation control solution and a quick response to the change as long as the system is observable.

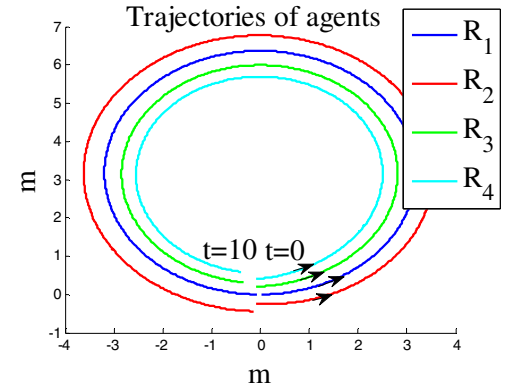

(a) Trajectories of agents in scenario 1

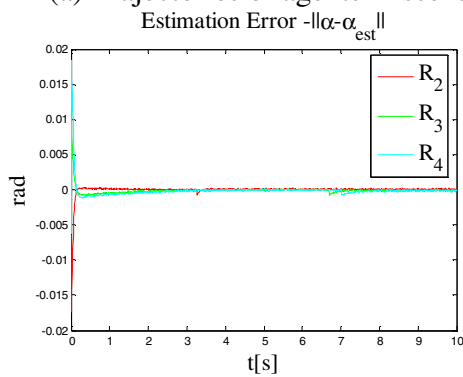

(c) Direction angle estimation error

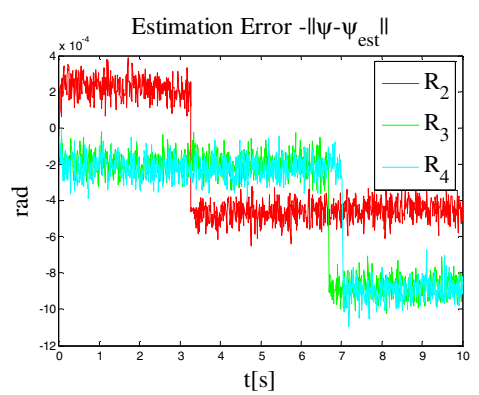

(b) Observation angle estimation error

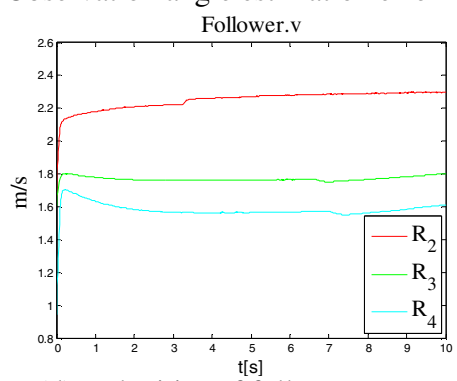

(d) Velocities of follower-agents

Fig. 3. Formation performance of scenario 1 


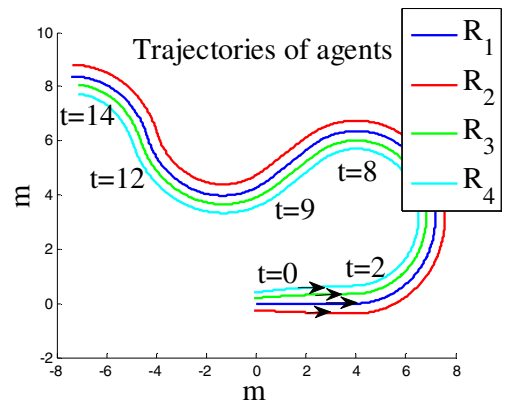

(a) Trajectories of agents in scenario 2

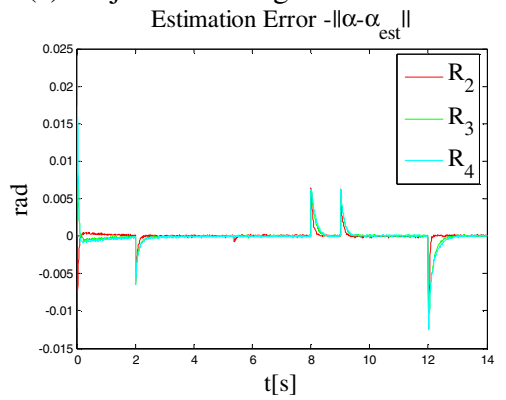

(c) Direction angle estimation error

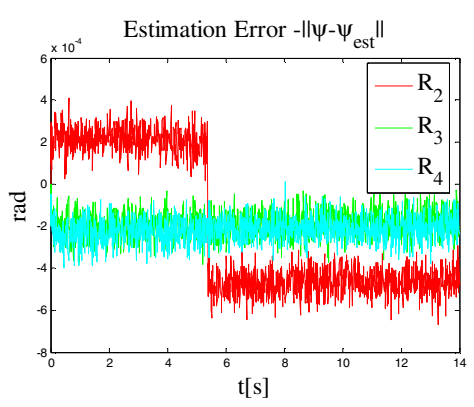

(b) Observation angle estimation error

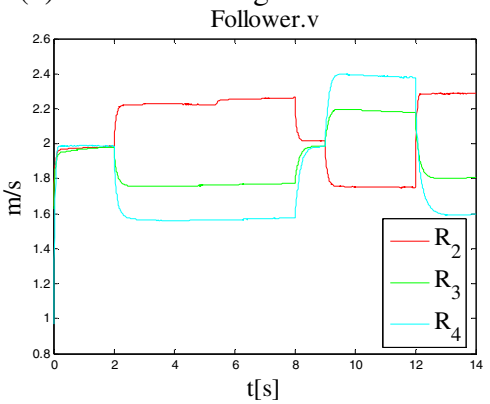

(d) Velocities of follower-agents

Fig. 4. Formation performance of scenario 2

\section{Conclusion}

A leader-follower formation control method is presented for multiple agents of the "1-N" structure where multiple followers follow one leader. The observability of the " $1-\mathrm{N}$ " leader-follower formation system is studied, which theoretically shows that the bearing-only observation meet the observability requirement if the leader and followers do not move straightly in parallel. The UKF is employed for the position estimation, which enables the movement control of the followers via input-output feedback control. Simulation results show that the system can rapidly obtain the desired formation and maintain the desired formation accurately and reliably. Further research for multi-agent formation control will consider dynamical obstacles.

Acknowledgements. This work was supported by the National Natural Science Foundation of China (Grant No.51475383, 51075337) and by the project Sociedades Humano-Agente en Entornos Cloud Computing (Soha+C) SA213U13.

\section{References}

1. Carrascosa, C., Bajo, J., Julián, V., Corchado, J.M., Botti, V.: Hybrid multi-agent architecture as a real-time problem-solving model. Expert Systems with Applications 34(1), 2-17 (2008) 
2. Zato, C., Villarrubia, G., Sánchez, A., Barri, I., Rubión, E., Fernández, A., Rebate, C., Cabo, J.A., Álamos, T., Sanz, J., Seco, J., Bajo, J., Corchado, J.M.: PANGEA - Platform for Automatic construction of organizations of intElligent Agents. In: 9th International Conference Distributed Computing and Artificial Intelligence (2012)

3. Ren, W., Beard, R.W.: Formation feedback control for multiple spacecraft via virtual structures. IEE Proceedings of the Control Theory and Applications, IET 151(3), 357-368 (2004)

4. Sun, T., Liu, F., Pei, H., He, Y.: Observer-based adaptive leader-following formation control for non-holonomic mobile robots. IET Control Theory \& Applications 6(18), 2835-2841 (2012)

5. Chen, J., Sun, D., Yang, J., Chen, H.Y.: A leader-follower formation control of multiple nonholonomic mobile robots incorporating receding-horizon scheme. The Int. J. Robotics Research 28, 727-747 (2009)

6. Shao, J., Xie, G., Wang, L.: Leader-following formation control of multiple mobile vehicles. IET Control Theory \& Applications 1(2), 545-552 (2007)

7. Consolini, L., Morbidi, F., Prattichizzo, D., Tosques, M.: Leader-follower formation control of nonholonomic mobile robots with input constraints. Automatica 44(5), 1343-1349 (2008)

8. Cristescu, S.M., Ionescu, C.M., Wyns, B., DeKeyser, R.: Leader-Follower String Formation using Cascade Control for Mobile Robots. In: 20th Mediterranean Conference on Control and Automation, pp. 1092-1098 (2012)

9. Kwon, J.W., Chwa, D.: Hierarchical formation control based on a vector field method for wheeled mobile robots. IEEE Tran. Robotics 28(6), 1335-1345 (2012)

10. Sharma, R., Beard, R.W., Taylor, C.N., Quebe, S.: Graph-based observability analysis of bearing-only cooperative localization. IEEE Transactions on Robotics 28(2), 522-529 (2012)

11. Moshtagh, N., Michael, N., Jadbabaie, A., Daniilidis, K.: Bearing-only control laws for balanced circular formations of ground robots. In: Proceedings of Robotics: Science and Systems IV. MIT Press (2008)

12. Basiri, M., Bishopand, A.N., Jensfelt, P.: Distributed control of triangular formations with angle-only constraints. Systems \& Control Letters 59(2), 147-154 (2010)

13. Bishop, A.N.: A very relaxed control law for bearing-only triangular formation control. In: Proceedings of the 18th IFAC World Congress, Milano, Italy, pp. 5991-5998 (2011a)

14. Bishop, A.N.: Distributed bearing-only quadrilateral formation control. In: Proceedings of the 18th IFAC World Congress, Milano, Italy, pp. 4507-4512 (2011b)

15. Eren, T.: Formation shape control based on bearing rigidity. International Journal of Control 85(9), 1361-1379 (2012)

16. Franchiand, A., Giordano, P.R.: Decentralized control of parallel rigid formations with direction constraints and bearing measurements. In: CDC, pp. 5310-5317 (2012)

17. Zhao, S., Lin, F., Peng, K., Chen, B.M., Lee, T.H.: Finite-time stabilisation of cyclic formations using bearing-only measurements. International Journal of Control 87(4), 715-727 (2014)

18. Zhao, S., Lin, F., Peng, K., Chen, B.M., Lee, T.H.: Distributed control of angleconstrained cyclic formations using bearing-only measurements. Systems \& Control Letters 63, 12-24 (2014)

19. Mariottini, G.L., Morbidi, F., Prattichizzo, D., et al.: Vision-based localization for leaderfollower formation control. IEEE Transactions on Robotics 25(6), 1431-1438 (2009)

20. Sharma, R., Quebe, S., Beard, R.W., Taylor, C.N.: Bearing-only Cooperative Localization. Journal of Intelligent \& Robotic Systems 72(3-4), 429-440 (2013) 
21. Li, T., Sun, S.: Double-resampling based Monte Carlo localization for mobile robot. Acta Automatica Sinica 36(9), 1279-1286 (2010)

22. Nerurkar, E.D., Roumeliotis, S.I., Martinelli, A.: Distributed maximum a posteriori estimation for multi-robot cooperative localization. In: Robotics and Automation, ICRA 2009, pp. 434-439 (2009)

23. Hermann, R., Krener, A.J.: Nonlinear controllability and observability. IEEE Transactions on Automatic Control 22(5), 728-740 (1977)

24. Morbidi, F., Marottini, G.L., Prattichizzo, D.: Observer design via Immersion and Invariance for vision-based leader-follower formation control. Automatica 46(1), 148-154 (2010)

25. Wan, E.A., Van Der Merwe, R.: The unscented Kalman filter for nonlinear estimation. In: The IEEE 2000 Adaptive Systems for Signal Processing, Communications, and Control Symposium 2000, AS-SPCC, pp. 153-158 (2000)

26. Slotine, J.J.E., Li, W.: Applied nonlinear control, vol. 46(1). Prentice-Hall, Englewood Cliffs (1991)

27. Das, A.K., Fierro, R., Kumar, V., et al.: A vision-based formation control framework. IEEE Transactions on Robotics and Automation 18(5), 813-825 (2002) 